\title{
DETERMINAÇÃO DE PARACETAMOL PELA INIBIÇÃO DA REAÇÃO QUIMILUMINESCENTE DO LUMINOL- HIPOCLORITO DE SÓDIO EM UM SISTEMA DE ANÁLISE EM FLUXO EMPREGANDO O CONCEITO DE MULTICOMUTAÇÃO
}

\author{
Geiser G. Oliveira, Bruno C. Janegitz, Marina B. Batistão, Fernanda H. Salami e Orlando Fatibello-Filho \\ Departamento de Química, Universidade Federal de São Carlos, CP 676, 13560-970 São Carlos - SP, Brasil \\ Oldair D. Leite*
}

Instituto de Ciências Ambientais e Desenvolvimento Sustentável, Universidade Federal da Bahia, 47805-100 Barreiras - BA, Brasil

Recebido em 4/8/08; aceito em 11/3/09; publicado na web em 4/8/09

\begin{abstract}
DETERMINATION OF PARACETAMOL USING A FLOW INJECTION ANALYSIS WITH MULTICOMMUTATION AND CHEMILUMINESCENCE DETECTION. A flow injection chemiluminescence method for the determination of paracetamol in pharmaceutical formulations is described. It is based on the consumption of the sodium hypochlorite by paracetamol and decreases of the analytical signal. The analytical curve was linear in the paracetamol concentration range from $5.0 \times 10^{-6}$ to $5.0 \times 10^{-5} \mathrm{~mol} \mathrm{~L}^{-1}$, with a detection limit of $1.8 \times 10^{-6} \mathrm{~mol} \mathrm{~L}^{-1}$. The RSDs were 2.0 and $1.2 \%$ respectively for $2.0 \times 10^{-5}$ and $4.0 \times 10^{-5} \mathrm{~mol} \mathrm{~L}^{-1}$ paracetamol solutions $(n=10)$ and a sampling frequency of $180 \mathrm{~h}^{-1}$ was obtained.
\end{abstract}

Keywords: paracetamol; chemiluminescence; flow injection analysis.

\section{INTRODUÇÃO}

Paracetamol (acetoaminofenol, $\mathrm{N}$-acetil- $p$-aminofenol, 4-acetoamidofenol), ${ }^{1}$ Figura 1 , é um fármaco com propriedades analgésicas e antipiréticas. Faz parte da composição de uma série de medicamentos usados contra a constipação comum e gripe. Introduzido por von Mering em 1893, este fármaco é o analgésico mais vendido no país, porém seu consumo inadequado pode causar problemas nas células hepáticas, sendo que seu uso deve ser evitado no caso de suspeita de dengue, principalmente na forma hemorrágica. ${ }^{2}$<smiles>CC(=O)Nc1ccc(O)cc1</smiles>

Figura 1. Estrutura molecular do paracetamol

Há na literatura vários procedimentos descritos para a determinação de paracetamol em formulações farmacêuticas e em fluidos biológicos, empregando técnicas cromatográficas, ${ }^{3,4}$ amperométricas ${ }^{5,6}$ e espectrofotométricas: $:^{1,7-12}$ (espectrometria de absorção molecular no visível), ${ }^{7-9}$ (quimiluminescência). ${ }^{1,10-12}$

Alguns trabalhos são descritos na literatura para determinação de analitos envolvendo a inibição da quimiluminescência. ${ }^{10-12}$ Alapont et al. ${ }^{10}$ desenvolveram um sistema de análise por injeção em fluxo para a determinação indireta de paracetamol empregando o sistema quimiluminescente luminol- $\mathrm{H}_{2} \mathrm{O}_{2}-\left[\mathrm{Fe}(\mathrm{CN})_{6}\right]^{3-}$. A curva analítica obtida foi linear entre $1,4 \times 10^{-5} \mathrm{e} 7,0 \times 10^{-5} \mathrm{~mol} \mathrm{~L}^{-1}$, sendo o limite de detecção igual a $1,1 \times 10^{-5} \mathrm{~mol} \mathrm{~L}^{-1}$. Nalewajko et al. ${ }^{11}$ desenvolveram um método de análise por injeção em fluxo para a determinação

*e-mail: oldair@ufba.br de dopamina em produtos farmacêuticos. Nesse método em fluxo foi explorada a inibição da intensidade da quimiluminescência da reação luminol-hexacianoferrato (III) em médio básico. Duas curvas analíticas foram obtidas nos intervalos de concentração do analito de $1,9 \times 10^{-7}$ a $6,5 \times 10^{-7} \mathrm{~mol} \mathrm{~L}{ }^{-1}$ e de $2,6 \times 10^{-6}$ a $1,9 \times 10^{-5} \mathrm{~mol} \mathrm{~L}^{-1}$, com limite de detecção de $3,3 \times 10^{-8} \mathrm{~mol} \mathrm{~L}^{-1}$. Para a determinação de carbaril, Waseem et al. ${ }^{12}$ desenvolveram um procedimento de análise por injeção em fluxo empregando-se a inibição da reação quimiluminescente entre luminol-cobalto(II)- $\mathrm{H}_{2} \mathrm{O}_{2}$ em meio alcalino pelo carbaril. A curva analítica foi linear no intervalo de concentração de carbaril de 5,0 x $10^{-7}$ a $20 \times 10^{-6} \mathrm{~mol} \mathrm{~L}^{-1}$, com um limite de detecção de $2,4 \times 10^{-7} \mathrm{~mol} \mathrm{~L}^{-1}$.

O sistema de análise por injeção em fluxo (FIA) com multicomutação é uma alternativa interessante para a análise química, apresentando baixo consumo de reagentes, minimizando a produção de resíduos, apresentando alta frequência de amostragem e boa versatilidade. ${ }^{9}$ Os procedimentos analíticos com detecção por quimiluminescência destacam-se por apresentar boa sensibilidade e ampla faixa de resposta linear. ${ }^{13}$ A quimiluminescência nada mais é que a produção de radiação luminosa por uma reação química. ${ }^{14} \mathrm{O}$ luminol é um reagente quimiluminescente, com reação conhecida desde 1928, comumente utilizado na determinação de diversos analitos. ${ }^{14-19}$

No presente trabalho, desenvolveu-se um sistema de análise em fluxo empregando-se o conceito de multicomutação para a determinação de paracetamol em formulações farmacêuticas empregando-se a inibição da magnitude do sinal gerado pela reação quimiluminescente entre luminol e hipoclorito, que foi proporcional ao aumento da concentração de paracetamol nas amostras.

\section{PARTE EXPERIMENTAL}

\section{Equipamentos}

O sistema de análise em fluxo empregando o conceito de multicomutação construído para a determinação de paracetamol é apresentado na Figura 2. Foram empregados válvulas solenoides de três vias (NResearch, West Caldwell, 161T031), tubos de polietileno (0,8 mm de d.i) e confluências feitas de acrílico. A propulsão dos fluidos foi 
realizada com o auxílio de uma bomba peristáltica Ismatec (IPC-12), equipada com tubos de propulsão de Tygon. O controle do módulo de análise e a aquisição dos dados foram realizados empregando-se um microcomputador Pentium de $800 \mathrm{MHz}$, equipado com uma interface eletrônica (Advantec Corp., PCL-711S). A linguagem de programação utilizada foi escrita em LabVIEW 6.0. As medidas da radiação quimiluminescente foram feitas empregando-se um fotodetector (OSD50-E, RS 846-711) 20 $^{20}$ uma cela de fluxo em polietileno. ${ }^{21}$ Mais detalhes do sistema de detecção podem ser obtidos na ref. 21.

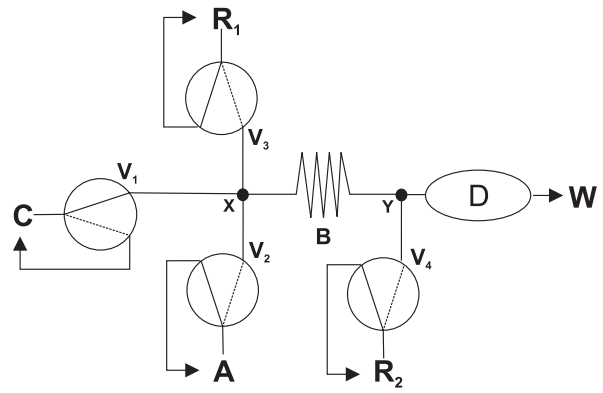

Figura 2. Diagrama de fluxos do sistema empregado para a determinação de paracetamol. $C$-transportador; A - soluções padrões de paracetamol el ou amostras; $R_{1}$ - solução de hipoclorito de sódio; $R_{2}$ - solução de luminol; $V_{1}-V_{4}$-válvulas solenoides de 3 vias; $B$ - bobina helicoidal; D-Detector: luminômetro construído com fotodiodo de silício contendo uma cela de polietileno em espiral de $100 \mathrm{~cm}$; W-descarte; X e Y-ponto de confluência

\section{Diagrama de fluxo e procedimento}

No módulo de análise apresentado na Figura 2 foram empregadas 4 válvulas solenoides $\left(\mathrm{V}_{1}-\mathrm{V}_{4}\right)$, responsáveis pelo gerenciamento das soluções dos reagentes, soluções padrões/amostras e do transportador. De acordo com a Figura 2, apenas o transportador água deionizada $\left(V_{1}\right)$ flui pelo percurso analítico, enquanto as soluções dos reagentes $\left(\mathrm{V}_{3} \mathrm{e}_{4}\right)$ e amostra $\left(\mathrm{V}_{2}\right)$ estão reciclando para os respectivos frascos. Inicialmente foi realizada a obtenção do sinal da luminescência da reação entre o hipoclorito de sódio e luminol (sinal do branco). Para a obtenção do branco, a válvula $\mathrm{V}_{3}$ (hipoclorito de sódio) é acionada, e juntamente com a solução transportadora, fluem pelo percurso analítico por um tempo pré-estabelecido. Na sequência, é então acionada a válvula $\mathrm{V}_{4}$ (luminol), por um tempo pré-estabelecido, permitindo a reação entre o luminol e o hipoclorito de sódio, obtendo-se assim o sinal da luminescência (sinal do branco). Na Figura 3a é apresentado um esquema da reação quimilminescente entre o hipoclorito de sódio e o luminol em meio alcalino.

Após a obtenção do sinal do branco, foi realizada a detecção da luminescência promovida pela reação entre o paracetamol, hipoclorito de sódio e luminol (Figura 3b). Neste procedimento, as válvulas solenoides $\mathrm{V}_{2}$ (paracetamol), $\mathrm{V}_{3}$ (hipoclorito de sódio) e a $\mathrm{V}_{1}$ (solução transportadora) são simultaneamente acionadas por um tempo préestabelecido. Assim, o transportador (água deionizada) $\left(\mathrm{V}_{1}\right)$ retorna para seu frasco e alíquotas de amostra/padrões $\left(\mathrm{V}_{2}\right)$ e solução de hipoclorito de sódio $\left(\mathrm{V}_{3}\right)$ são inseridas no percurso analítico no ponto X. Na bobina helicoidal (B), ocorre então a reação entre a solução de paracetamol e hipoclorito de sódio (oxidação do paracetamol pelo hipoclorito). $\mathrm{Na}$ sequência, a válvula $\mathrm{V}_{4}$ é então acionada e uma alíquota de luminol é inserida no sistema no ponto Y. O luminol reage com o excesso de hipoclorito, que não foi consumido pelo paracetamol, obtendo-se assim o sinal da luminescência. A diferença entre os sinais do branco (hipoclorito e luminol) e da reação entre hipoclorito, paracetamol e luminol (supressão da luminescência) é determinada, sendo esta proporcional à concentração de paracetamol na amostra ou na solução de referência. A Figura $3 b$ apresenta a reação do hipoclorito de sódio com paracetamol.

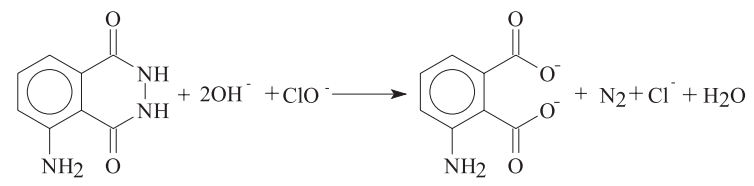<smiles>CC(=O)Nc1cccc(O)c1</smiles>

Figura 3. (a) Reação quimiluminescente entre o luminol e o hipoclorito de sódio e (b) reação entre hipoclorito e paracetamol, onde há diminuição da magnitude do sinal analítico

\section{Reagentes e soluções}

As soluções foram preparadas empregando-se água desionizada e reagentes de grau analítico. Foi preparada uma solução estoque de luminol (Aldrich) 9,0 mmol L-1 em solução de $\mathrm{K}_{2} \mathrm{CO}_{3}$ (Aldrich) $0,60 \mathrm{~mol} \mathrm{~L}^{-1} \mathrm{com} \mathrm{pH} 11$. A solução estoque de hipoclorito de sódio (Vetec) $0,10 \mathrm{~mol} \mathrm{~L}^{-1}$ foi diariamente preparada a partir de uma solução de $6 \%$ v/v e a solução de paracetamol (Aldrich) na concentração de $0,01 \mathrm{~mol} \mathrm{~L}^{-1}$ foi preparada para posterior diluição apropriada em água desionizada.

\section{Preparo das amostras}

Dez comprimidos foram exatamente pesados e triturados com auxílio de um almofariz e pistilo. Uma massa exatamente pesada de paracetamol foi transferida para um balão volumétrico de 100 $\mathrm{mL}$ e dissolvida com água deionizada. A solução foi filtrada e uma alíquota desta solução transferida para um balão volumétrico de 25 mL e completado com água deionizada. A solução obtida apresentou concentração dentro da faixa linear, sendo então empregada para a determinação de paracetamol empregando-se o sistema em fluxo proposto.

\section{RESULTADOS E DISCUSSÃO}

O procedimento analítico proposto baseia-se no consumo do reagente hipoclorito de sódio pelo paracetamol, sendo o excesso de hipoclorito de sódio determinado por quimiluminescência com luminol e o seu decréscimo relacionado com a concentração de paracetamol na amostra do fármaco (Figura 3).

$\mathrm{Na}$ otimização do sistema por injeção em fluxo com multicomutação foi empregado o método univariado com o propósito de se obter os melhores parâmetros analíticos do sistema em fluxo e também das soluções empregadas.

\section{Efeito da concentração da solução de hipoclorito de sódio}

Inicialmente, estudou-se o efeito da concentração de hipoclorito de sódio no intervalo de concentração de 0,50 a $2,5 \mathrm{mmol} \mathrm{L}^{-1}$ sobre o sinal analítico, utilizando o sistema mostrado na Figura 2. Neste estudo as seguintes condições foram empregadas: transportador água desionizada, $66 \mu \mathrm{L}$ de solução de paracetamol 1,5 x 10 $0^{-5} \mathrm{~mol} \mathrm{~L}^{-1}\left(\mathrm{~V}_{2}\right.$ 
$=2 \mathrm{~s}), 66 \mu \mathrm{L}$ de solução de hipoclorito de sódio $\left(\mathrm{V}_{3}=2 \mathrm{~s}\right)$ e $66 \mu \mathrm{L}$ de solução de luminol 1,0 mmol L-1 $\left(\mathrm{V}_{4}=2 \mathrm{~s}\right)$, bobina helicoidal de $25 \mathrm{~cm}$ e vazão de $2,0 \mathrm{~mL} \mathrm{~min}^{-1}$. Todas as medidas foram feitas em triplicata. Observou-se um aumento do sinal analítico nas concentrações entre 0,50 a $1,0 \mathrm{mmol} \mathrm{L}^{-1}$. Acima dessa concentração não houve um aumento significativo da variação do sinal analítico, sendo então selecionada a solução de hipoclorito de sódio com concentração de $1,0 \mathrm{mmol} \mathrm{L}^{-1}$ para os estudos posteriores.

\section{Efeito da concentração da solução de luminol}

Estudou-se o efeito das concentrações de luminol no intervalo de concentração de 0,50 a 4,0 $\mathrm{mmol} \mathrm{L}^{-1}$, mantendo-se as condições do sistema fixadas em: $66 \mu \mathrm{L}$ de solução de paracetamol $1,5 \times 10^{-5}$ $\mathrm{mol} \mathrm{L}^{-1}\left(\mathrm{~V}_{2}=2 \mathrm{~s}\right), 66 \mu \mathrm{L}$ de solução hipoclorito de sódio $1,0 \mathrm{mmol}$ $\mathrm{L}^{-1}\left(\mathrm{~V}_{3}=2 \mathrm{~s}\right)$ e $66 \mu \mathrm{L}$ de solução de luminol $\left(\mathrm{V}_{4}=2 \mathrm{~s}\right)$, bobina helicoidal de $25 \mathrm{~cm}$ e vazão de $2,0 \mathrm{~mL} \mathrm{~min}^{-1}$. Todas as medidas foram feitas em triplicata.

Um aumento significativo do sinal analítico foi observado entre as concentrações de 0,05 e 2,0 $\mathrm{mmol} \mathrm{L}^{-1}$ (Figura 4). Acima desta concentração a variação do sinal analítico praticamente permaneceu constante, Portanto, a concentração de luminol $2,0 \mathrm{mmol} \mathrm{L}^{-1}$ foi selecionada para os estudos futuros.

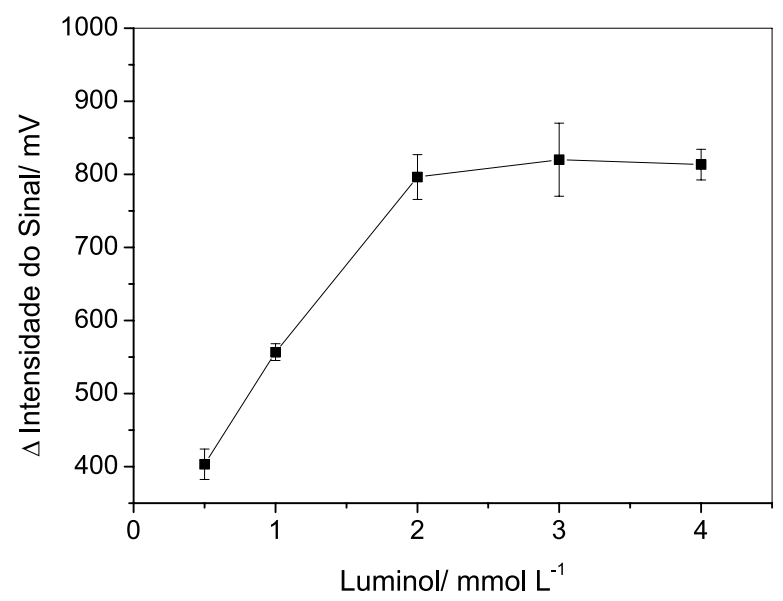

Figura 4. Efeito da concentração de luminol sobre o sinal analítico nas seguintes condições: $66 \mu L$ de solução de paracetamol $1,5 \times 10^{-5} \mathrm{~mol} \mathrm{~L}^{-1}$ $\left(V_{2}=2 \mathrm{~s}\right), 66 \mu \mathrm{L}$ de solução hipoclorito de sódio $1,0 \mathrm{mmol} L^{-1}\left(V_{3}=2 \mathrm{~s}\right) \mathrm{e}$ $66 \mu \mathrm{L}$ de solução de luminol $\left(V_{4}=2 \mathrm{~s}\right)$, bobina helicoidal de $25 \mathrm{~cm}$ e a uma de vazão 2,0 $\mathrm{mL} \mathrm{min}^{-1}$

\section{Estudo do tempo de acionamento das válvulas das soluções de hipoclorito de sódio e paracetamol}

Estudou-se a variação dos volumes do sistema reacional no sistema em fluxo. Nesta otimização, as soluções de hipoclorito de sódio e de paracetamol foram avaliadas simultaneamente, variando-se o tempo de acionamento das válvulas solenoides entre 2 e $5 \mathrm{~s}$. Neste estudo, a vazão de todos os canais, transportador e reagentes, foi fixada em 2,0 $\mathrm{mL}$ min $^{-1}$. As demais condições empregadas nestes estudos são descritas a seguir: transportador água deionizada, solução de paracetamol a 1,5 x $10^{-5} \mathrm{~mol} \mathrm{~L}^{-1}$, solução de hipoclorito de sódio $1,0 \mathrm{mmol} \mathrm{L}^{-1}, 66 \mu \mathrm{L}$ de solução de luminol $\left(\mathrm{V}_{4}=2 \mathrm{~s}\right)$ e bobina helicoidal de $25 \mathrm{~cm}$. Neste estudo, observou-se um aumento do sinal analítico com o tempo de acionamento das válvulas solenoides $\mathrm{V}_{2} \mathrm{eV}_{3}$ até $3 \mathrm{~s}$, mantendo-se constante nos tempos de acionamento superiores. Assim, um tempo de $3 \mathrm{~s}$ de acionamento das válvulas foi selecionado para os estudos posteriores.

\section{Estudo do tempo de acionamento da válvula da solução de} luminol

Avaliou-se o efeito do tempo de acionamento da válvula solenoide $\mathrm{V}_{4}$ (luminol), entre 2 e $5 \mathrm{~s}$. Observou-se que há um aumento do sinal analítico entre 2 e $3 \mathrm{~s}$ do acionamento da mesma. Acima de 3 s, o sinal analítico praticamente se manteve constante. Provavelmente esta concentração de luminol é suficiente para reagir com o excesso de hipoclorito de sódio, que não reagiu com o paracetamol. $\mathrm{O}$ tempo de $3 \mathrm{~s}$ para o acionamento da válvula $\mathrm{V}_{4}$ (luminol) foi então selecionado.

\section{Avaliação do comprimento da bobina helicoidal}

Estudou-se o efeito do comprimento da bobina helicoidal (B) de 15 a $100 \mathrm{~cm}$. Neste estudo, observou-se que o maior sinal analítico foi obtido em um comprimento de $25 \mathrm{~cm}$; abaixo deste comprimento houve uma diminuição considerável da reprodutibilidade dos sinais analíticos e, acima desse valor, houve diminuição do sinal analítico, indicando assim um aumento na dispersão da zona de amostra. Optouse então em utilizar o comprimento de $25 \mathrm{~cm}$ da bobina helicoidal (B) para os estudos posteriores.

\section{Estudo da vazão do sistema}

Estudou-se o efeito da vazão das soluções simultaneamente para todas as soluções na faixa de 1,4 a 3,0 $\mathrm{mL} \mathrm{min}^{-1}$. Verificou-se um aumento no sinal analítico com a vazão até $2,0 \mathrm{~mL} \mathrm{~min}^{-1}$. Observou-se que acima desta vazão houve o aumento na pressão hidrodinâmica do sistema, além de não se obterem sinais reprodutíveis. A vazão de $2,0 \mathrm{~mL} \mathrm{~min}{ }^{-1}$ foi selecionada por apresentar boa estabilidade e repetibilidade dos sinais analíticos obtidos.

\section{Estudo dos interferentes em potencial}

Avaliou-se o efeito dos interferentes em potencial na determinação de paracetamol em formulações farmacêuticas. Normalmente nestas formulações estão presentes além do paracetamol, excipientes e estabilizantes como amido, cafeína, celulose e estearato de sódio.

Nesses experimentos empregou-se uma solução de referência de paracetamol de $1,5 \times 10^{-5} \mathrm{~mol} \mathrm{~L}^{-1}$ juntamente com cada um dos interferentes em potencial nas concentrações declaradas nos produtos comerciais. Os resultados obtidos nestes estudos mostraram que não houve interferência significativa sobre o sinal analítico nos teores estudados para essas substâncias.

\section{Curva analítica e aplicação}

Após a otimização dos parâmetros físico-quimicos do procedimento em fluxo, a curva analítica apresentou uma linearidade no intervalo de concentração de paracetamol de 5,0 x $10^{-6}$ a 5,0 × $10^{-5}$ mol L $\mathrm{L}^{-1}$ como mostrada na Figura 5, sendo descrita pela equação: Sinal $(\mathrm{mV})=2,18-2,84 \times 10^{4}$ [Paracetamol] $\left(\mathrm{mol} \mathrm{L}^{-1}\right),(\mathrm{r}=0,999)$. Um limite de detecção $\left.(\mathrm{LD}=3 \times \mathrm{x} \sigma) / 2,84 \times 10^{4}\right)$ de $1,8 \times 10^{-6} \mathrm{~mol} \mathrm{~L}^{-1}$ foi obtido.

Estudou-se também a repetibilidade do procedimento em fluxo proposto. Os RSDs foram iguais a 2,0 e 1,2\%, respectivamente, para soluções de referência de paracetamol 2,0 x $10^{-5}$ e 4,0 x 10-5 $\mathrm{mol} \mathrm{L}^{-1}(\mathrm{n}=$ 10). Uma frequência de amostragem de $180 \mathrm{~h}^{-1}$ foi obtida neste sistema em fluxo, com baixo consumo de reagentes por determinação (24,0 $\mu \mathrm{g}$ de luminol e 7,5 $\mu \mathrm{g}$ de hipoclorito de sódio por determinação).

Os resultados obtidos no estudo de adição e recuperação do analito em três diferentes concentrações de paracetamol, adicionadas a 


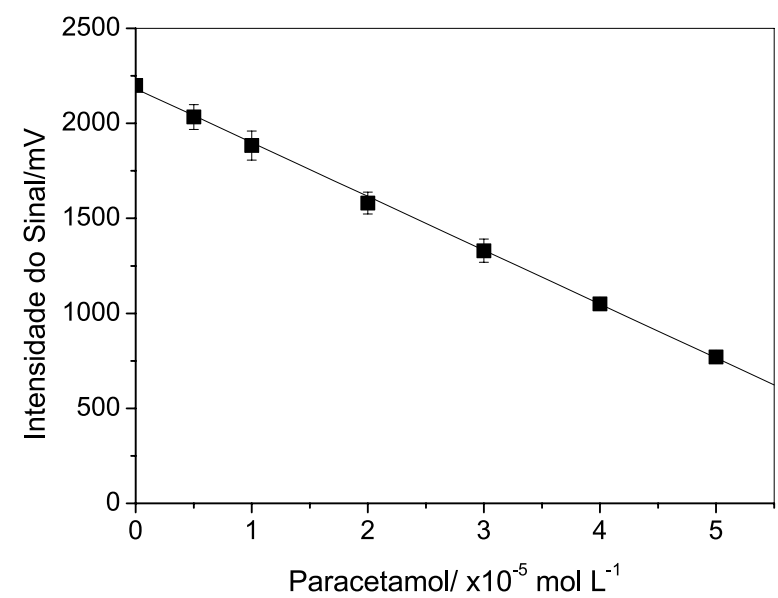

Figura 5. Curva analítica obtida para as soluções padrões de paracetamol de 5,0 $\times 10^{-6}$ a 5,0 $\times 10^{-4} \mathrm{~mol} \mathrm{~L}^{-1}$

três diferentes amostras de formulações farmacêuticas são mostrados na Tabela 1. Como pode ser observado, as recuperações de paracetamol variaram de 98,0 a 103\%, evidenciando que não há interferência significativa da matriz da amostra.

Tabela 1. Estudo de adição e recuperação de paracetamol em amostras farmacêuticas

\begin{tabular}{lccc}
\hline Amostras & $\begin{array}{c}\text { Adicionado } \\
\left(\times 10^{-5} \mathrm{~mol} \mathrm{~L}^{-1}\right)\end{array}$ & $\begin{array}{c}\text { Recuperado } \\
\left(\times 10^{-5} \mathrm{~mol} \mathrm{~L}^{-1}\right)\end{array}$ & $\begin{array}{c}\text { Recuperação } \\
(\%)\end{array}$ \\
\hline A & 1,00 & $1,02 \pm 0,03$ & 101 \\
& 1,00 & $1,00 \pm 0,02$ & 100 \\
B & 1,00 & $1,03 \pm 0,02$ & 103 \\
& 3,00 & $3,00 \pm 0,05$ & 100 \\
C & 3,00 & $3,00 \pm 0,05$ & 100 \\
& 3,00 & $2,94 \pm 0,07$ & 98,0 \\
\hline
\end{tabular}

O método proposto foi aplicado na determinação de paracetamol em formulações farmacêuticas e comparado com o método espectrofotométrico da Farmacopéia Americana. ${ }^{21}$ A Tabela 2 apresenta os resultados obtidos empregando-se o método proposto e o comparativo.

Empregando-se o teste-t pareado aos resultados obtidos dos teores de paracetamol nos produtos farmacêuticos empregando-se o método proposto e, os teores obtidos, empregando-se o método da
Farmacopéia Americana, encontrou-se uma boa concordância entre resultados a um nível de confiança de $95 \%$, estando os erros relativos dentro de um intervalo aceitável.

As figuras de mérito obtidas neste trabalho e aquelas apresentadas em trabalhos descritos na literatura para a determinação de paracetamol são mostradas na Tabela 3.

A comparação das figuras de mérito do procedimento proposto com aqueles apresentados nos trabalhos de Alapont et al. ${ }^{10} \mathrm{e}$ Ruengsitagoon et al., ${ }^{1}$ verifica-se uma maior faixa de resposta linear no procedimento proposto por Ruengsitagoon et al., ${ }^{1}$ no entanto, os três procedimentos apresentaram sensibilidades analíticas similares. Entretanto, cabe salientar que os módulos de detecção empregados naqueles sistemas analíticos se basearam em fotomultiplicadoras, dispositivo eletrônico mais sensível e, consequentemente, muito mais caro, que o fotodiodo de silício, empregado no trabalho aqui proposto. Ademais, uma comparação do consumo de reagentes (no caso luminol) do trabalho de Alapont et al. ${ }^{10}$ onde foi empregado um sistema de análise por injeção fluxo clássico, com o consumo no trabalho proposto (multicomutação), estimou um gasto de 170 $\mu \mathrm{g}$ de luminol/determinação contra $24 \mu \mathrm{g}$ de luminol/determinação, respectivamente (dados não disponibilizados na Tabela 3).

Todos os métodos espectrofotométricos se destacam pela ampla faixa linear das curvas analíticas que, de certa forma, facilita a diluição da amostra quando necessária. Outrossim, só há um artigo descrito na literatura com a mesma frequência de amostragem que o método proposto $\left(180 \mathrm{~h}^{-1}\right)$, muito superior que as demais frequências analíticas apresentadas nos outros artigos.

Os procedimentos com detecção amperométrica destacam-se pela ampla faixa linear da curva analítica e, também, pela economia de reagentes. No artigo de Cervini et al. ${ }^{6}$ destaca-se também a frequência de amostragem igual ao procedimento proposto. $\mathrm{O}$ mérito do procedimento aqui proposto neste caso se reflete na simplicidade do sistema de detecção utilizado.

\section{CONCLUSÕES}

O procedimento analítico em fluxo com detecção quimiluminescente proposto para a determinação de paracetamol em formulações farmacêuticas apresentou-se preciso na quantificação do mesmo.

Os resultados obtidos neste procedimento foram concordantes com aqueles obtidos no método comparativo. Com o emprego das válvulas solenoides para o gerenciamento das soluções no sistema envolvendo multicomutação, verificaram-se um baixo consumo de reagentes, boa sensibilidade e linearidade da curva analítica e uma alta frequência de amostragem.

Tabela 2. Determinação de paracetamol em produtos farmacêuticos comerciais pelo método quimiluminescente e pelo método comparativo ${ }^{20}$

\begin{tabular}{|c|c|c|c|c|c|}
\hline \multirow{2}{*}{ Amostras } & \multicolumn{3}{|c|}{ Paracetamol (mg g $\left.{ }^{-1}\right)$} & \multicolumn{2}{|c|}{$\operatorname{Er}(\%)$} \\
\hline & Valor rotulado & Comparativo & Proposto & $\mathrm{E}_{1}$ & $\mathrm{E}_{2}$ \\
\hline A & 750 & $750 \pm 2$ & $749 \pm 3$ & $-0,1$ & $-0,1$ \\
\hline B & 750 & $751 \pm 3$ & $750 \pm 2$ & 0 & $-0,1$ \\
\hline $\mathrm{C}$ & 750 & $750 \pm 1$ & $751 \pm 4$ & $+0,1$ & $+0,1$ \\
\hline $\mathrm{D}$ & 750 & $750 \pm 2$ & $752 \pm 3$ & $+0,3$ & $+0,3$ \\
\hline $\mathrm{E}$ & 500 & $501 \pm 3$ & $502 \pm 2$ & $+0,4$ & $+0,2$ \\
\hline $\mathrm{F}$ & 500 & $499 \pm 4$ & $500 \pm 1$ & 0 & $+0,2$ \\
\hline G & 500 & $500 \pm 1$ & $500 \pm 2$ & 0 & 0 \\
\hline $\mathrm{H}$ & 500 & $501 \pm 1$ & $499 \pm 2$ & $-0,2$ & $-0,4$ \\
\hline
\end{tabular}

$\mathrm{n}=3$, nível de confiança de $95 \%$. $\mathrm{E}_{1}=$ procedimento proposto $v s$ valor rotulado. $\mathrm{E}_{2}=$ procedimento proposto $v s$ procedimento comparativo. 
Tabela 3. Procedimentos analíticos em fluxo para a determinação de paracetamol

\begin{tabular}{|c|c|c|c|c|c|}
\hline Método & $\begin{array}{l}\text { Linearidade } \\
(\mu \mathrm{mol} \mathrm{L}-1)\end{array}$ & $\begin{array}{c}\mathrm{LD} \\
\left.(\mu \mathrm{mol} \mathrm{L})^{-1}\right)\end{array}$ & $\begin{array}{c}\text { Frequência } \\
\text { de amostragem } \mathrm{h}^{-1}\end{array}$ & $\begin{array}{l}\mathrm{CV} \\
(\%)\end{array}$ & Ref. \\
\hline Quimiluminescência * & 5,0 a 50 & 1,8 & 180 & $2,0(n=10)$ & Proposto \\
\hline Quimiluminescência ${ }^{\#}$ & 14 a 70 & 10 & 87 & $2,2(n=25)$ & 10 \\
\hline Quimiluminescência\$ & 2,0 a 282 & 1,0 & 90 & $1,1(n=20)$ & 1 \\
\hline Espectrofotométrico & 8,50 a 250 & 5,0 & 60 & $1,2(\mathrm{n}=10)$ & 7 \\
\hline Espectrofotométrico & 3,30 a 660 & 1,6 & 80 & $<1,0(\mathrm{n}=10)$ & 8 \\
\hline Espectrofotométrico & 28,2 a 705 & 2,2 & 60 & $1,5(n=11)$ & 9 \\
\hline Amperométrico & 282,2 a 1350 & 1,3 & 60 & N.A & 5 \\
\hline Amperométrico & 50,0 a 5000 & 20 & 180 & N.A & 6 \\
\hline
\end{tabular}

*Inibição da quimiluminescência da reação entre luminol e hipoclorito de sódio; \#inibição da quimiluminescência da reação entre luminol/ peróxido de hidrogênio/ferricianeto de potássio; ${ }^{\$}$ quimiluminescência gerada na reação entre um complexo de rutênio (III), permanganato de sódio e paracetamol, na presença de íons manganês

\section{AGRADECIMENTOS}

Ao CNPq, à CAPES e FAPESP pelo suporte financeiro.

\section{REFERÊNCIAS}

1. Ruengsitagoon, W.; Liawruangrath, S.; Townshend, A.; Talanta 2006, $69,976$.

2. www.anvisa.gov.br/farmacovigilancia/informes/2002/informe_2.htm acessada em Julho 2008.

3. Budimir, N.; Weston, D. J.; Creaser, C. S.; Analyst 2007, 132, 34.

4. Nebot, C.; Gibb, S. W.; Boyd, K. G.; Anal. Chim. Acta 2007, 598, 87.

5. Santos, W. T. P.; Almeida, E. G. N.; Ferreira, H. E. A.; Gimenes, D. T.; Richter, E. M.; Electroanalysis 2008, 20,1878.

6. Cervini, P.; Cavalheiro, E. T. G.; J. Braz. Chem. Soc. 2008, 19, 836.

7. Fatibello-Filho, O.; Vieira, H. J.; Ecl. Quim. 2008, 33, 47.

8. Aniceto, C.; Fatibello-Filho, O.; Quim. Nova 2002, 25, 387.

9. Lavorante, A. F.; Feres, M. A.; Reis, B. F.; Spectr. Lett. 2006, 39, 631.

10. Alapont, A. G.; Zamora, L. L.; Calatayud, J. M.; J. Pharm. Biomed. Anal. 1999, 21, 311.
11. Nalewajko, E.; Ramirez, R. B.; Kojlo, A.; J. Pharm. Biomed. Anal. 2004, $36,219$.

12. Waseem, A.; Yaqoob, M.; Nabi, A.; Siddiqui, M. A.; Int. J. Environ. Anal. Chem. 2007, 87, 825.

13. Borges, E. P.; Fernandes, E. N.; Rocha, F. R. P.; Reis, B. F.; Quim. Nova 2002, 25, 1191 .

14. Ferreira, E. C.; Rossi, A. V.; Quim. Nova 2002, 25, 1003.

15. Li, Y. H.; Tang, Y. H.; Yao, H.; Fu, J. M.; Luminescence 2003, 18, 313.

16. Lau, C. W.; Qin, X. J.; Liang, H. Y.; Lu, H. Z.; Anal. Chim. Acta 2004, $514,45$.

17. Economou, A.; Themelis, D. G.; Theodoridis, G.; Tzanavaras, P. D.; Anal. Chim. Acta 2002, 463, 249.

18. Wang, L.; Yang, P.; Li, Y. X.; Zhu, C. Q.; Talanta 2006, 70, 219.

19. Pan, J.; Huang, Y. M.; Shu, W. Q.; Cao, J.; Talanta 2007, 71, 1861.

20. Leite, O. D.; Fatibello-Filho, O.; Vieira, H. J.; Rocha, F. R. P.; de Miranda Cury, N. S.; Anal. Lett. 2007, 40, 3148.

21. Leite, O. D.; Fatibello-Filho, O.; Rocha, F. R. P.; Quim. Nova 2004, 27, 337.

22. The United States Pharmacopeia, $24^{\text {th }}$ ed.; Rockville, 2000. 